\title{
Motor Unit Function in Skeletal Muscle Autografts of Rats
}

\author{
Claude Côté AND JoHn A. FAUlKNer \\ Department of Physiology, The University of Michigan Medical School, \\ Ann Arbor, Michigan 48109
}

Received August 5, 1983; revision received November 22, 1983

\begin{abstract}
Standard and nerve-intact grafts of the extensor digitorum longus (EDL) muscles of rats do not fully recover control values for maximum tetanic tension $\left(P_{o}\right)$. We compared the physiologic properties of motor units from standard and nerve-intact EDL grafts of rats with those of control EDL muscles. Standard grafts were completely removed and replaced in their original site. For nerve-intact grafts all physical connections were severed except for the nerve. Isometric contractile properties of whole muscles and single motor units were measured in situ 75 and 50 days after surgery for standard and nerve-intact grafts, respectively. Motor units from both types of grafts showed a mean and distribution for time-to-peak twitch tension (TPT) not different from control motor units. The absolute $P_{o}$ of nerve-intact grafts showed a greater recovery than the standard grafts, but was still significantly lower than the control value. The average decrease in the $P_{o}$ of motor units from nerve-intact grafts of $16 \%$ was not different from the decrease in the $P_{o}$ of the total EDL graft which suggests no loss of motor units in nerve-intact grafts. In standard grafts, the $57 \%$ decrease in $P_{0}$ for the whole muscle was attributable to a $20 \%$ decrease in the average tension development per motor unit and a $45 \%$ decrease in the number of motor units. These differences suggest a more complete reinnervation of the nerve-intact grafts than standard grafts.
\end{abstract}

\section{INTRODUCTION}

Successful regeneration of fibers in free whole skeletal muscle autografts in rats relies on the spontaneous revascularization and reinnervation of the ischemic and denervated tissue. In standard grafts, no attempt is made to repair the severed nerve. To evaluate the role of reinnervation on the regeneration of skeletal muscle fibers, Carlson et al. (4) developed a grafting procedure in which the motor nerve was left intact. Studies of standard and

Abbreviations: EDL-extensor digitorum longus, $\mathrm{P}$-tension, $\mathrm{P}_{\mathrm{o}}$-maximum isometric tetanic tension, TPT-time-to-peak twitch tension, $P_{t}$-peak twitch tension, FF-fast-fatigable, FR-fast-fatigue resistant, FI-fast intermediate, S-slow. 
nerve-intact grafts have focused on the restoration of structure and function of whole grafts $(4,10)$. Compared with control extensor digitorum longus (EDL) muscles, the maximum isometric tetanic tension $\left(\mathrm{P}_{\mathrm{o}}\right)$ developed by standard EDL grafts varies between 40 and $50 \%(4,11)$ whereas that of nerveintact grafts may attain $90 \%$ (4). In both standard and nerve-intact grafts the total number of fibers is not significantly different from control muscles (4).

No information is available on the restoration of structure and function of single motor units in regenerated muscles. The present experiments characterized the function of single motor units in standard and nerve-intact grafts of EDL muscles of rats. We hypothesized that the major difference in $P_{0}$ between standard grafts and control EDL muscles was due to both a decrease in the number of motor units per muscle and a decrease in the average $P_{o}$ of motor units whereas the small difference between the $P_{o}$ of nerve-intact grafts and control muscles resulted solely from a reduced average $\mathbf{P}_{\mathrm{o}}$ per motor unit. We described the contractile properties of the three traditional types of motor units found in the control EDL muscles and postulated that the average time-to-peak twitch tension and resistance to fatigue of single motor units would not be significantly different whether the motor unit was situated in a graft or control EDL muscle.

\section{MATERIALS AND METHODS}

Data were obtained on motor units from grafts and control EDL muscles of 65 female Sprague-Dawley rats. The mean weight of the rats was $250 \pm 10 \mathrm{~g}$. All rats were exposed to a 12:12 light:dark cycle and given food and water ad libitum. Control data were obtained on 30 rats and the remaining 35 rats were assigned to experimental groups.

Surgical Procedures. For operative procedures and measurements of contractile properties, rats were anesthetized by i.p. injection of sodium pentobarbital $(50 \mathrm{mg} / \mathrm{kg})$, followed by approximately one-quarter of the initial dose every 1 to $2 \mathrm{~h}$. In 15 rats, the EDL muscle was grafted orthotopically using standard procedures. In 20 other rats the EDL muscle was grafted with the motor nerve intact. Only one EDL muscle was grafted in each animal. The standard procedure involved the transection of the proximal and distal tendons and all nerves and blood vessels to the EDL muscle. The muscle was then removed and replaced in its original site. The tendons were sutured to the stumps of the original tendons but revascularization and reinnervation occurred spontaneously. Grafts with nerves intact were obtained in a similar manner with the exception that the major nerve branches innervating the EDL muscle were not severed. Care was taken to disrupt the small blood vessels accompanying the nerve branches. After grafting, the portion of the nerves within the nerve-intact grafts undergoes degeneration and regeneration (4). 
Contractile Properties. Contractile properties of single motor units were measured at $80 \pm 3$ and $52 \pm 2$ days after grafting, for standard and nerveintact grafts, respectively. Those periods of recovery were selected because previous studies demonstrated that a variety of morphologic, physiologic, and biochemical characteristics have stabilized in these types of grafts by this time (4). Our goal was to study motor unit characteristics only in stabilized grafts. To isolate the muscle or graft, the skin covering the hind limb was first cut around the ankle and then pulled up to the level of the upper thigh. The EDL muscle or graft was dissected free and the distal tendon was cut. Care was taken not to damage the vascular and nerve supplies. A metal hook was tied to the distal tendon of the muscle or graft for attachment to a force transducer.

With the rat in the prone position, the hind limb was placed in a bath through an opening in one wall. The bath was sealed by stretching the skin over a Plexiglas ring surrounding the hole and tying it in position. The knee and the ankle were rigidly fixed with metal pins. The bath contained approximately $200 \mathrm{ml}$ mineral oil maintained at $37 \pm 1^{\circ} \mathrm{C}$.

Isometric contractions of the whole EDL muscle or graft and of single motor units were elicited with supramaximal square pulses lasting $0.2 \mathrm{~ms}$. The force transducer, (Kulite Semiconductor Products, Model BG-100) was fastened to a rack and pinion device permitting adjustment of muscle length and tension. Muscle length was adjusted to give maximum twitch tension of the whole muscle. This optimal length $\left(L_{o}\right)$ was held constant for all subsequent measurements. Force development during isometric contractions was analyzed by a computer and plotted on an X-Y digital plotter. Tensions between 0.05 and $250 \mathrm{~g}$ could be recorded reliably. Experiments were terminated when the $P_{o}$ of the whole muscle was found to be less than $90 \%$ of the original value. In $95 \%$ of cases, the muscles, and therefore the motor units, showed little change in tension during the course of the experiments which lasted between 8 and $10 \mathrm{~h}$. Peak twitch tension $\left(P_{t}\right)$, time-to-peak twitch tension (TPT), half-relaxation time, the frequency-force relationship, and the maximum isometric tetanic tension $\left(P_{o}\right)$ were first recorded for the whole muscle (6). Those same variables were later obtained for each motor unit that was isolated. Single motor units were also characterized for their resistance to fatigue by stimulating each unit for $200 \mathrm{~ms}$ each second at 80 $\mathrm{Hz}$. The time required for the tension to decline to one-half the initial value was used as an index of fatigability. The maximum duration of the fatigue test was set at $1000 \mathrm{~s}$.

When the electrical activity of the contracting motor units was recorded, a fine, stainless-steel, monopolar needle was implanted in the EDL muscle or graft. The signal was amplified using a Grass PGAC amplifier and the 
resulting action potential visualized on a storage oscilloscope. Two methods of stimulation were used to elicit contractions of single motor units. In some animals, single motor units were stimulated through the ventral root filaments. In this case, the left hind limb was denervated by transecting the femoral and obturator nerves and all branches of the sciatic nerve except the branches of the common peroneal nerve innervating the EDL muscle. Axons of motor units in rat EDL muscles have been shown to be distributed equally in L4 and $L 5$ ventral roots. These roots were exposed by laminectomy and transected proximally near the spinal cord. The skin around the incision over the vertebral column was drawn up to form a pool of mineral oil also at $37^{\circ} \mathrm{C}$. The roots were dissected under a microscope until only one motor axon supplying the EDL muscle was found to be on the electrodes.

In other cases, single motor units were stimulated through motor axons of the common peroneal nerve. The whole sciatic nerve was exposed and the common peroneal branch dissected from the rest of the nerve; that branch was cut at the level of the hip so that the total length of free nerve available for dissection was approximately 3 to $4 \mathrm{~cm}$. All muscles innervated by that branch of the sciatic nerve were denervated except for the EDL muscle. The nerve branch was kept in the same mineral oil bath used for the limb. At that point, the fascia covering the nerve was removed and nerve filaments were subdivided progressively until they contained only one axon to the EDL muscle. This technique is comparable to that used by Smith and Lännergren (17). No differences were observed between data collected by the two different methods of stimulation.

In both types of preparations the criteria for a single motor unit were allor-nothing responses of the mechanical and electrical activities of a muscle or graft to finely graded stimuli from subthreshold to supramaximal current strength and pulse duration. If during the characterization of a single unit the mechanical or electrical tracing showed any evidence of a second motor unit firing sporadically, the stimulation protocol was terminated and the dissection was resumed.

Single motor units were classified using the physiologic classification proposed by Burke et al., (2) but adapted to the faster contracting rat muscle. This classification is based on the sensitivity to fatigue of motor units and on the presence or absence of "SAG" during unfused tetanic contractions. Burke et al. (2) defined SAG as the decline in tension development observed immediately after attainment of maximum tension during unfused tetanic contractions. Fast-twitch motor units invariably showed SAG whereas slowtwitch motor units never showed SAG. Using the criteria of SAG and fatigability, single motor units were typed as slow (S), fast-fatigable, (FF), or fast-fatigue resistant (FR). In this study, fast units with fatigue times of less 
than $100 \mathrm{~s}$ were classified as FF units and the FR typing was ascribed to units with fatigue times greater than $250 \mathrm{~s}$; units with values between 100 and $250 \mathrm{~s}$ were referred to as fast intermediate units (FI).

Statistical Tests. A one-way analysis of variance was used to compare the data from the three groups of muscles. When the $F$ ratio was significant, group means were compared with the Duncan multiple range test.

\section{RESULTS}

The contractile properties of control muscles, standard, and nerve-intact grafts are presented in Table 1. The mean wet weight of standard grafts was $80 \%$ of that of control EDL muscles and this difference was found to be statistically significant. No significant difference was found between the muscle weight of nerve-intact grafts and control muscles.

The mean absolute $P_{0}$ for control EDL muscles was $1.67 \pm 0.1 \mathrm{~N}$. For standard and nerve-intact grafts, the absolute $\mathrm{P}_{\mathrm{o}}$ was 43 and $80 \%$ of the control value, respectively. When normalized for the total cross-sectional area, $P_{v}$ was $21.4 \mathrm{~N} / \mathrm{cm}^{2}$ in control EDL muscles and $9.3 \mathrm{~N} / \mathrm{cm}^{2}$ and 16.3 $\mathrm{N} / \mathrm{cm}^{2}$ for standard and nerve-intact grafts, respectively. All these differences were statistically significant.

\section{TABLE 1}

Comparison of Isometric Contractile Properties of Autografts to Control Extensor Digitorum Longus Muscles ${ }^{a}$

\begin{tabular}{|c|c|c|c|c|c|c|}
\hline \multirow[b]{3}{*}{ Muscle mass (g) } & & & \multicolumn{4}{|c|}{ Autografts } \\
\hline & \multicolumn{2}{|c|}{$\begin{array}{c}\text { Control } \\
(N=35)^{b}\end{array}$} & \multicolumn{2}{|c|}{$\begin{array}{l}\text { Standard } \\
(N=11)\end{array}$} & \multicolumn{2}{|c|}{$\begin{array}{l}\text { Nerve-intact } \\
(N=18)\end{array}$} \\
\hline & 157.6 & \pm 8.4 & 126.7 & $\pm 13.1^{*}$ & 154.7 & \pm 9.8 \\
\hline $\begin{array}{l}\text { Time-to-peak } \\
\text { tension }(\mathrm{ms})\end{array}$ & 11.9 & \pm 0.2 & 12.4 & \pm 0.3 & 12.0 & \pm 0.5 \\
\hline $\begin{array}{l}\text { 1/2 Relaxation time } \\
\text { (ms) }\end{array}$ & 10.6 & \pm 0.3 & 12.1 & $\pm 1.0^{*}$ & 12.4 & $\pm 0.6^{* *}$ \\
\hline $\begin{array}{l}\text { Maximum isometric } \\
\text { tension }(\mathrm{N})\end{array}$ & 1.67 & \pm 0.09 & 0.71 & $\pm 0.10^{* *}$ & 1.31 & $\pm 0.09^{* *, * * *}$ \\
\hline $\begin{array}{l}\text { Maximum isometric } \\
\text { tension }\left(\mathrm{N} / \mathrm{cm}^{2}\right)\end{array}$ & 21.4 & \pm 1.1 & 9.3 & $\pm 1.2^{* *}$ & 16.3 & $\pm 0.9^{*, * * * *}$ \\
\hline $\begin{array}{r}\text { Twitch:tetanus } \\
\text { tension ratio }\end{array}$ & 0.184 & \pm 0.009 & 0.10 & $\pm 0.009^{* *}$ & 0.13 & $\pm 0.009^{* *, * * * *}$ \\
\hline
\end{tabular}

a Data are group means $\pm \mathrm{SE}$.

${ }^{b}$ Number of muscles used.

* Significantly different from control value $(P<0.05)$.

** Significantly different from control value $(P<0.01)$.

**** Significantly different from standard graft value $(P<0.01)$. 
The values for TPT for each of the three groups were not significantly different with an average value close to $12.0 \mathrm{~ms}$. Half-relaxation time was found to be significantly longer in both groups of autografts compared with $10.6 \pm 0.3 \mathrm{~ms}$ obtained for control EDL muscles. A twitch:tetanus ratio of $0.18 \pm 0.01$ was calculated for control muscles. The values of $0.10 \pm .01$ obtained for standard grafts and $0.14 \pm .01$ for nerve-intact grafts were significantly smaller than the control value.

Characteristics of Motor Units. The distribution of absolute $P_{0}$ values for motor units of control muscles and of standard and nerve-intact grafts is shown in Fig. 1. Control EDL muscles showed a major distribution peak between 10 and $30 \mathrm{mN}$ and a second peak between 40 and $60 \mathrm{mN}$. Some motor units had $P_{o}$ values as high as 100 to $120 \mathrm{mN}$. Compared to a value of $9 \%$ for control EDL muscles, more than $40 \%$ of the motor units in standard grafts had values for $P_{o}$ equal or less than $10 \mathrm{mN}$. The distribution for standard grafts, unlike that for nerve-intact grafts, covered the same range of absolute $P_{o}$ values as control EDL muscles. Some motor units in standard

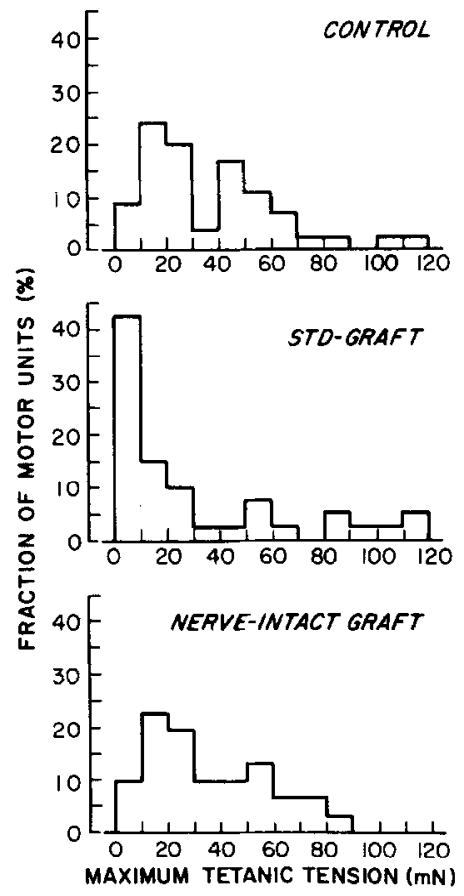

FIG. 1. Distribution of the absolute maximum isometric tetanic tension for control extensor digitorum longus (EDL) muscles, standard, and nerve-intact grafts. Data from 86 motor units from control EDL muscles, 45 motor units from standard EDL grafts, and 36 motor units from nerve-intact EDL grafts. 
grafts produced tetanic tensions up to $120 \mathrm{mN}$. Nerve-intact grafts, by comparison, had a larger proportion of their motor units with $P_{o}$ values between 10 and $30 \mathrm{mN}$. The peak observed between 40 and $60 \mathrm{mN}$ for control muscles was absent in nerve-intact grafts as were the units with very large $P_{o}$ values. The mean absolute $P_{n}$ for motor units from control EDL muscles was $38 \pm 3.6 \mathrm{mN}$. Mean absolute $\mathrm{P}_{\mathrm{o}}$ 's were 79 and $84 \%$ of the control value for motor units of standard and nerve-intact grafts, respectively.

The range of relative tensions within the muscles is more clearly shown when the $\mathbf{P}_{\mathrm{o}}$ of motor units is expressed as a percentage of the whole muscle $P_{o}$ (Fig. 2). The distribution for standard grafts differed from that of the other two. Most motor units of standard grafts were found under one single large peak between 0 and $4 \% P / P_{o}$ whereas the largest units reached relative tensions of $14 \%$. Control muscles and nerve-intact grafts had very similar distributions with respect to the shape and range of the histogram.

The distributions of values for TPT in control EDL muscles, standard, and nerve-intact grafts were very similar (Fig. 3). These distributions had the same relative shape and extended over the same range. By interrelating the physiologic properties of motor units, a three-dimensional physiologic profile can be obtained. Figure 4 shows the profile for control EDL muscles. Type

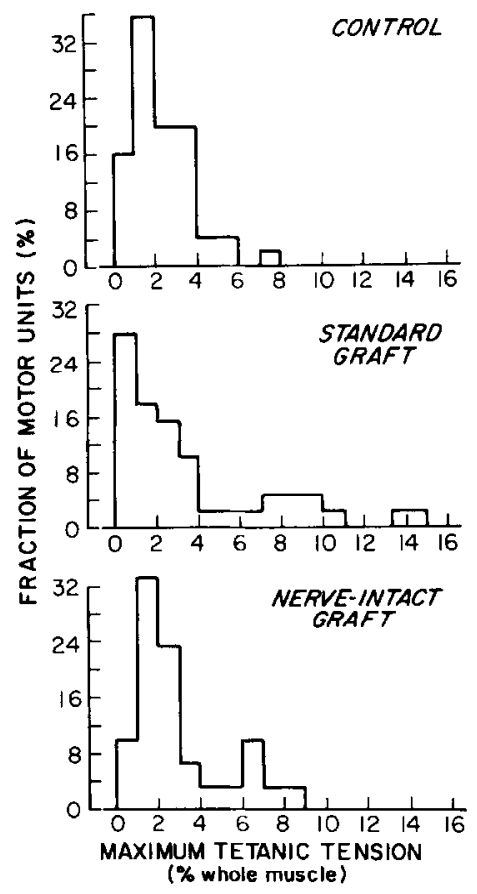

Fig. 2. Distribution of the maximum isometric tetanic tension $\left(P_{0}\right)$ for the same motor units showed in Fig. 1 plotted as percentage of $P_{o}$ for the whole muscle. 

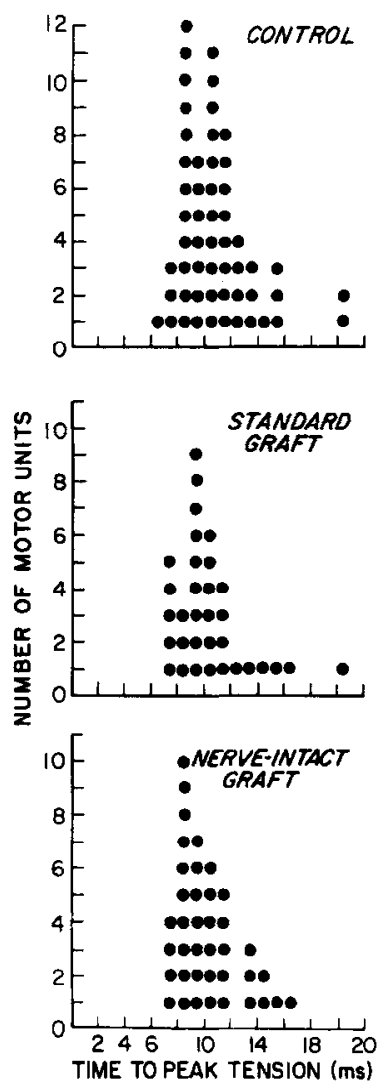

FIG. 3. Distribution of isometric twitch time-to-peak tension for EDL motor units from control muscles $(N=55)$, standard $(N=33)$, and nerve-intact grafts $(N=39)$. Data obtained at $37^{\circ} \mathrm{C}$ with muscle length adjusted to produce maximum twitch tension of the whole muscle.

FF units were easily identified because they formed a cluster with low values for fatigue index and TPT and large values for $P_{0}$. Type $S$ units are identified by a long TPT, low values for $P_{0}$, and no evidence of SAG. The distribution of fatigue indices was continuous between the FF and FR type units. High fatigue indices were associated with low $P_{0}$ 's, whether the unit was of type $S$ or FR. With this classification, fast units formed a continuum of values for TPT ranging from 6.8 to $16 \mathrm{~ms}$.

The physiologic profile of standard grafts (Fig. 5) was similar to that of control EDL muscles. However, relatively more motor units were classified as FF units than in the control muscles. Nerve-intact grafts showed some motor units with a relatively long TPT and a low fatigue index (Fig. 6). In control muscles, a high value for TPT was always associated with a high fatigue index. 


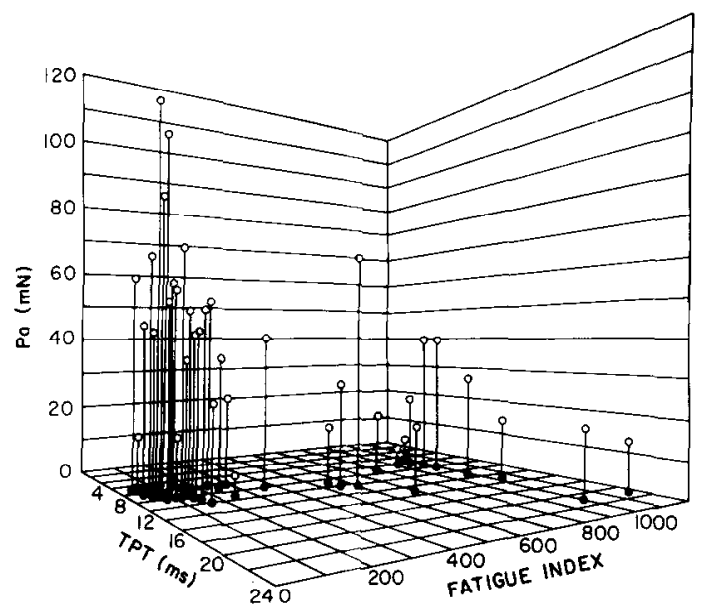

F1G. 4. Three-dimensional diagram illustrating the profile of motor units for control EDL muscles $(N=45)$. Increasing values of fatigue index denote increasing resistance to fatigue during a standardizcd tetanic stimulation protocol. Each line, bound by black and white circles, indicales an individual motor unit. Time-to-peak twitch tension (TPT) and fatigue index are indicated on the ordinate and abscissa. The height of the line is the maximal isometric tetanic tension $\left(\mathbf{P}_{\mathrm{o}}\right)$.

The average number of motor units per muscle can be estimated by dividing the average $P_{o}$ of whole muscles by the mean $P_{o}$ of motor units. This method, when used with a sample of motor units representative of the whole population of motor units, gave results similar to those obtained by the counting of the alpha-motoneurons (15). The number of motor units estimated for control

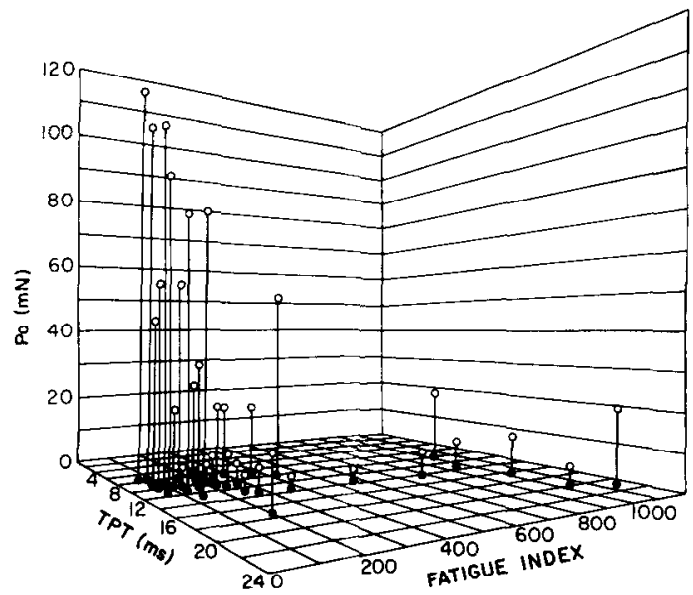

FIG. 5. Profile of motor units for standard EDL grafts $(N=33)$. Note the appearance of a large number of very small motor units. Same legend as Fig. 4. 
EDL muscles was 44. Nerve-intact grafts had an almost identical value of 41 and an average of only 24 motor units per muscle was found for the standard grafts.

The relative proportions of the different motor unit types in control EDL muscle and both types of grafts are shown in Table 2. Significant differences in motor unit composition were observed among the three groups. Whereas $36 \%$ of the control motor units were classified as FR units, $64 \%$ of the motor units in nerve-intact grafts were of the FR type. In spite of differences in motor unit composition, no significant difference was observed among the three groups for the average fatigue index for motor units.

\section{DISCUSSION}

The EDL muscle of rats has been studied extensively as the classic fasttwitch muscle in comparison with the slow-twitch soleus muscle $(6,7,16)$. For EDL muscles studied in situ, values for TPT range from 11 to $12 \mathrm{~ms}$ $(6,7)$ and values for half-relaxation time from 8 to $11 \mathrm{~ms}(4,6,7)$. Our values for TPT and half-relaxation time for control EDL muscles are in good

TABLE 2

Number and Percentage of Types of Motor Units in Autografts Compared with Control Extensor Digitorum Longus Muscles

\begin{tabular}{|c|c|c|c|}
\hline & \multirow[b]{2}{*}{$\begin{array}{l}\text { Control } \\
(N=86)^{a}\end{array}$} & \multicolumn{2}{|c|}{ Autografts } \\
\hline & & $\begin{array}{l}\text { Standard } \\
(N=45)\end{array}$ & $\begin{array}{l}\text { Nerve-intact } \\
(N=36)\end{array}$ \\
\hline $\begin{array}{l}{ }^{b} \text { Average motor unit maximum } \\
\text { tetanic tension }(\mathrm{mN})\end{array}$ & $38.0 \pm 3.6$ & $30.2 \pm 5.4^{*}$ & $32.1 \pm 4.3^{*}$ \\
\hline $\begin{array}{l}\text { 'Average motor unit fatigue } \\
\text { time (s) }\end{array}$ & $394.1 \pm 68.4$ & $346.8 \pm 61.4$ & $452.5 \pm 76.1$ \\
\hline $\begin{array}{l}\text { ' Estimated number of motor } \\
\text { units per muscle }\end{array}$ & 44 & 24 & 41 \\
\hline$\%$ FR units ${ }^{d}$ & 36 & 30 & 64 \\
\hline$\%$ FF units & 38 & 24 & 18 \\
\hline$\%$ FI units & 21 & 34 & 13 \\
\hline$\% \mathrm{~S}$ units & 5 & 12 & 5 \\
\hline
\end{tabular}

${ }^{a}$ Number of motor units characterized.

${ }^{b}$ Data are group means $\pm \mathrm{SE}$.

${ }^{c}$ Mean whole muscle $P_{0}$ divided by mean motor unit $P_{0}$. By using only the muscles or grafts where we were able to characterize at least five motor units, the estimates for the number of motor units per muscle were $47 \pm 7$ for control muscles, $21 \pm 4$ for standard grafts, and $42 \pm 4$ for nerve-intact grafts $(\bar{x} \pm \mathrm{SE})$. The difference between control muscles and standard grafts was significant $(P<0.01)$.

${ }^{d}$ FR-fast-fatigue resistant, FF-fast fatigable, FI-fast-intermediate, $S$-slow.

- Significantly different from control value $(P<0.02)$. 
agreement with these values. Similarly, our value of 0.18 for twitch:tetanus ratio of control EDL muscles is not significantly different from the value of 0.19 reported by Close $(6,7)$.

When $P_{0}$ is normalized per $\mathrm{cm}^{2}$ of cross-sectional area, values ranging from 15 to $30 \mathrm{~N} / \mathrm{cm}^{2}$ have been reported for various mammalian muscles (8). Our value of $21.4 \pm 0.9 \mathrm{~N} / \mathrm{cm}^{2}$ for control EDL muscles is in the middle range of these data. Our value for normalized $P_{0}$ was obtained at the end of long experiments during which many maximum contractions were elicited. Because repeated contractions induce significant weight gains compared with nonactive muscles (14), our value for normalized $P_{o}$ is an underestimation. Faulkner et al. (11) reported a mean weight gain of $20 \%$ after repeated contractions by EDL muscles of cats. A correction for a $20 \%$ weight gain would increase the normalized $P_{o}$ of the EDL muscles to approximately $26 \mathrm{~N} / \mathrm{cm}^{2}$.

Our distribution of values for TPT of motor units from control EDL muscles of rats is similar to that of Close (7). Both the shape of the histogram, with the main peak occurring between 9 and $11 \mathrm{~ms}$, and the range of TPT values observed are similar. Like Close, we found only two motor units with values of TPT more than $20 \mathrm{~ms}$. This observation agrees with the data on histochemical fiber typing which classifies $2 \%$ of the fibers in EDL muscle of rat as slow (1).

Values of $P_{0}$ for control motor units were found to be distributed in the range of 0 to $8 \%$ of the $P_{o}$ of the whole muscle. Close (7) reported a similar histogram for the distribution of $P_{o}$ values. By dividing the average $P_{o}$ for whole muscles by the average $P_{0}$ for motor units, Close estimated the number of motor units present in control EDL muscles of rat to be approximately 40. Our value of 44 motor units per control EDL muscle is in reasonable agreement with that value. Based on contraction times and tension development of whole muscles and single motor units, we conclude that our data for control EDL muscles agree with published results for the contractile properties of control EDL muscles and motor units of rats measured in situ. Therefore, our sample of motor units appears to be representative of the whole population of units.

In a comparison of standard and nerve-intact grafts, Carlson et al. (4) in rats and Faulkner et al. (11) in cats reported that the presence of an intact nerve significantly improved the functional recovery of muscle autografts. In rats the average $P_{o}$ of the nerve-intact grafts was $91 \%$ of the control value and standard grafts $57 \%$. Our values of 84 and $42 \%$ for nerve-intact and standard grafts arc in good agrecment with those values. Twitch tension and the twitch:tetanus ratio also recovered to a greater extent in the nerve-intact grafts than in the standard grafts.

The normalized $P_{o}$ 's of autografts werc 9.3 and $16.3 \mathrm{~N} / \mathrm{cm}^{2}$, respectively, for standard and nerve-intact autografts. These values are significantly lower than the $21.4 \mathrm{~N} / \mathrm{cm}^{2}$ obtained for control muscles. Skeletal muscle grafts 


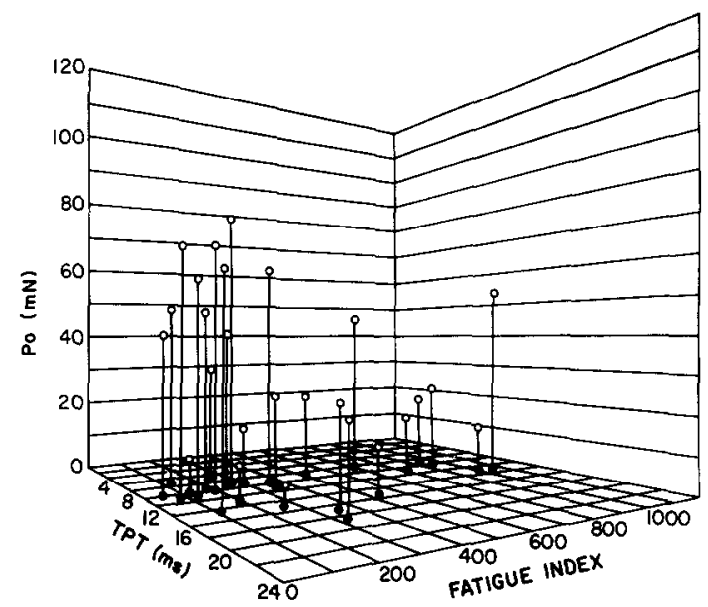

FIG. 6. Profile of motor units for nerve-intact EDL grafts $(N=26)$. Motor units with relatively slow time-to-peak tension values and low fatigue indices were found. Same legend as Fig. 4.

have increased amounts of connective tissue compared with control muscles $(4,9,11,12)$. Based on an increased mass of connective tissue in grafts, a significantly lower value for $P_{o}$ normalized for the total cross-sectional area was expected.

Carlson et al. (4) found that both TPT and half-relaxation time were not significantly different from control values in both standard and nerve-intact grafts. Our findings for TPT values of standard and nerve-intact grafts support this observation. The small but significantly prolonged values for half-relaxation time may be attributed to mechanical factors (5). These observations are consistent with previous reports that regenerated skeletal muscle fibers have normal contractile properties (11). The differences observed between regenerated muscle in grafts and control muscles result from the smaller viable cross-sectional area of grafts and the larger proportion of connective tissue (4).

Some aspects of the motor unit distribution of grafts differed from those of control EDL muscles. Standard grafts were composed of a few abnormally large motor units and a large number of small motor units. The number of motor units per muscle was estimated at only 24 in standard grafts whereas nerve-intact grafts contained $\mathbf{4 1}$ motor units. These findings support our hypotheses concerning the decreased maximum tension development in autografts. Nerve-intact grafts have approximately the same total number of motor units as control EDL muscles, but on the average each motor unit produces less tension. For standard grafts, the reduced $P_{0}$ results from a decreased number of motor units and significantly smaller tension development per motor unit. Faulkner $e$ t al. (11) reported that the specific tension 
$\left(\mathrm{N} / \mathrm{cm}^{2}\right)$ of grafts was not different from that of control muscles. Therefore, the maximum tension development of a motor unit of a graft is dependent on the number of muscle fibers composing the unit and on the cross-sectional area of the muscle fibers. Because the total number of muscle fibers in stabilized standard and nerve-intact EDL grafts of the rat is unchanged compared to control muscle (4), the decreased average $P_{o}$ of motor units in grafts is the result of a decrease in the mean fiber cross-sectional area.

Most differences between standard and nerve-intact grafts may be attributed to differences in the process of reinnervation. Whereas in the nerve-intact grafts nerve fibers regenerate through the old nerve channels, nerve fibers in standard grafts must grow through the endomysial connective tissue before reaching the regenerating muscle fibers (4). Our data on the number of motor units in standard grafts support the hypothesis that some nerve fibers do not find a passage through such a barrier.

In motor units of the gastrocnemius muscle of the cat, Burke et al. (2) found a clear division between the type FF and FR units in terms of resistance to fatigue. Motor units of skeletal muscles of rats, unlike motor units of cat skeletal muscles, have not been characterized as regards their resistance to fatigue. Although our fatigue index was obtained with a higher firing frequency adapted to the faster contraction of EDL muscles of rats compared with cats, a comparison with the data of Burke et al. is valid. Our data show that in control EDL muscles and in grafts there is a continuum of values for fatigue indices ranging from the low values of type FF units to the high values of the type $S$ units. Other studies have also observed such a continuum $(3,18)$.

The motor unit profiles of both standard and nerve-intact grafts were different from that of control EDL muscles. In control muscles, slow TPT values were associated with high fatigue indices, whereas in the nerve-intact grafts motor units with relatively slow TPT values (16 to $18 \mathrm{~ms})$ and low fatigue indices were found. Those units can be compared with the type IB fibers (slow-fatigable) that can be found in skeletal muscles of rat after a variety of operative procedures (13). Even though numerous hypotheses have been postulated, the basic mechanism underlying the status of these fibers remains obscure.

In summary, the decrease in $P_{o}$ observed in stabilized standard and nerveintact grafts is consistent with modifications in the populations of motor units. The average motor unit $P_{o}$ was decreased in both the standard and nerve-intact grafts. In addition, standard grafts showed a decrease in the total number of motor units. The differences between the populations of motor units of standard and nerve-intact grafts can be attributed to incomplete reinnervation of standard grafts.

\section{REFERENCES}

1. ARIANO, M. A., R. B. ARMSTRONG, AND V. R. EDGERTON. 1973. Hindlimb muscle fiber populations of five mammals. J. Histochem. Cytochem. 21: 51-55. 
2. Burke, R. E., D. N. Levine, P. Tsairis, AND F. E. Zajac. 1973. Physiological types and histochemical profiles in motor units of the cat gastrocnemius. J. Physiol. (London) 234: 723-748.

3. BURKE, R. E., AND P. TSAIRIS. 1974. The correlation of physiological properties with histochemical characteristics in single muscle units. Ann. N.Y. Acad. Sci. 228: 145-159.

4. Carlson, B. M., P. HNIK, S. Tucek, R. Vejsada, D. M. Bader, and J. A. Faulkner. 1981. Comparison between grafts with intact nerves and standard free grafts of the rat extensor digitorum longus muscle. Physiol. Bohemoslov. 30: 505-514.

5. CTSKE, P. E. 1982. Chronic stimulation of extensor digitorum longus autografts in rats. Doctoral Dissertation, The University of Michigan.

6. ClOSE, R. 1964. Dynamic properties of fast and slow skeletal muscles of the rat during development. J. Physiol. (London) 173: 74-95.

7. CLOSE, R. 1967. Properties of motor units in fast and slow skeletal muscles of the rat. $J$. Physiol. (London) 193: 45-55.

8. Close, R. I. 1972. Dynamic properties of mammalian skeletal muscles. Physiol. Rev. 52: 129-197.

9. COAN, M. R., AND R. J. TOMANEK. 1981. The growth of regenerating soleus muscle transplants after ablation of the gastrocnemius muscle. Exp. Neurol. 71: 278-294.

10. FAUlKNeR, J. A., L. C. MAXWELL, T. P. WHITE, AND J. H. NIEMEYER. 1979. Characteristics of autografted mammalian skeletal muscles. Pages 485-492 in A. MAURO, Ed., Muscle Regeneration. Raven Press, New York.

11. Faulkner, J. A., J. H. Niemeyer, L. C. Maxwell, and T. P. White. 1980. Contractile properties of transplanted extensor digitorum longus muscles of cats. Am. J. Physiol. 238: C120-C126.

12. Faulkner, J. A., J. M. Markley, JR., K. K. McCully, C. R. Watters, and T. P. WHITE. 1983. Characteristics of cat skeletal muscles grafted with intact nerves or with anastomosed nerves. Exp. Neurol. 80: 682-696.

13. MUNTENER, M. 1982. A rapid and reversible muscle fiber transformation in the rat. Exp. Neurol. 77: 668-678.

14. MurPhy, R. A., AND A. C. BeARDSLey. 1974. Mechanical properties of the cat soleus muscle in situ. Am. J. Physiol. 227: 1008-1013.

15. PeYronNard, J. M., AND L. Charron. 1980. Muscle reorganization after partial denervation and reinnervation. Muscle Nerve 3: 509-518.

16. Ranatunga, K. W. 1982. Temperature-dependence of shortening velocity and rate of isometric tension development in rat skeletal muscle. J. Physiol. (London) 329: 465-483.

17. SMITH, R. S., AND J. LÄNNERGREN. 1968. Types of motor units in the skeletal muscle of "Xenopus Laevis." Nature (London) 217: 281-283.

18. Stephens, J. A., R. L. Gerlach, R. M. Reinking, and D. G. Stuart. 1973. Fatigabilities of medial gastrocnemius motor units in the cat. Pages 179-185 in R. B. STEIN, Ed., Control of Posture and Locomotion. Plenum, New York. 